AGUdo RUIZ, A., «La gratuidad de la actuación judicial de los representantes en el sistema de las legis actiones», REDUR 9,

diciembre 20II, págs. 203-215. ISSN I695-078X

\title{
LA GRATUIDAD DE LA ACTUACIÓN JUDICIAL DE LOS REPRESENTANTES EN EL SISTEMA DE LAS LEGIS ACTIONES
}

\author{
Alfonso AGUdo RuIZ \\ CATEDRÁTICO DE DERECHO ROMANO \\ UNIVERSIDAD DE LA RIOJA
}

SUMARIO: I. Los representantes procesales en el sistema de las legis actiones: pro populo, pro libertate, pro tutela y ex lege Hostilia. II. El cognitor: su existencia y su esfera de actividad en época de las legis actiones. III. Bibliografía. IV. Abreviaturas.

RESUMEN: El objetivo del presente estudio es el análisis de aquellos casos en que no era posible, material o jurídicamente, la presencia personal de alguna de las partes en las actuaciones procesales, constituyendo una excepción a la regla de la inadmisibilidad de la representación directa en el procedimiento de las legis actiones, según las noticias que nos suministra Justiniano en sus Instituciones (4. Io pr.). Igualmente, hemos abordado la polémica cuestión doctrinal de sí el cognitor existía ya en el primer procedimiento que conoció Roma, tal como nosotros sostenemos y, en su caso, su ámbito de actuación. En todos los casos anteriores la actuación procesal del representante se realiza gratuitamente.

THE GRATUITOUSNESS OF THE JUDICIAL INTERVENTION OF THE REPRESENTATIVES IN THE LEGIS ACTIONES PROCEDURE

ABSTRACT: The aim of this article is to analyze the cases in which the personal presence of one of the parties in the trial was not physical or juridically posible, which is an exception to the rule of the irreceivability of the direct representation in the procedure of the legis actiones as shown by Iustinian in the Institutions (4. Io pr.). We also deal with the polemical doctrinal subject of the existence of the cognitor in the first procedure used in Rome, such as we maintain, as well as the scope of operation. In all the previous cases, the judicial intervention of the representative is free.

Palabras ClAVES-Keywords: pro populo, pro libertate, pro tutela, ex lege Hostilia, cognitor.

I. Los representantes procesales en el sistema de las legis actiones: pro populo, pro libertate, pro tutela y ex lege Hostilia

Distinto de los jurisconsultos, abogados $u$ oradores que realizan funciones de asesoramiento o defensa de las partes en la contienda judicial, es la labor del representante de las mismas cuya actuación supone una aplicación al proceso, serie de actos sucesivos necesarios para 
la defensa legítima de los derechos, de la teoría general de la representación en los negocios jurídicos.

En la dogmática civil y procesal moderna, suele distinguirse dos clases de representación: la representación directa, inmediata o perfecta y la indirecta, mediata o imperfecta. Se entiende por representación directa aquélla en la que los efectos de los actos jurídicos, negocios o procesos realizados por el representante, constando su condición de tal, se transmiten directa e inmediatamente al representado hasta el punto de que se considera que quien realiza el negocio o el proceso es el dominus negotii o dominus litis. Por el contrario, se llama representación indirecta aquélla en donde los efectos se dan exclusivamente a favor y en contra del representante, pero éste, en virtud de la relación jurídica preexistente con su representado, está obligado a transmitir o trasladar los efectos jurídicos a la persona por cuenta de la cual o en cuyo interés actúa. En el primer caso, se dice que el representante obra por cuenta y en nombre del representado; en el segundo, por cuenta del representado y en nombre propio.

Arias Ramos resume así las varias causas que se han apuntado por la doctrina para explicar esta carencia: «Unas de índole teórica o doctrinal: repugnancia de la mentalidad romana, por su acendrado sentimiento de dignidad personal, a admitir que la actividad de una persona aprovechase a otra; el particular concepto de la obligación contractual romana como un vínculo exclusivo entre los que contratan; el formalismo de los modos de obligarse en el antiguo Derecho romano incompatible con la representación. Otras explicaciones miran más bien a circunstancias de ambiente social que determinaron el que no se sintiesen en Roma las necesidades o dificultades que la representación satisface o soluciona. La organización familiar, los medios auxiliares de irradiación de su actividad que para los negocios del pater familias suponían hijos, esclavos y personas in mancipio; la sencillez de la economía y el comercio primitivos; el uso frecuentísimo del nuntius; son motivos de ordinario recordados para explicar lo que se ha llamado el principio romano de la no representación» $(1938,46)$.

Unánimemente la doctrina romanística afirma que durante el período de vigencia del procedimiento de las legis acciones las partes habían de comparecer personalmente y desarrollar su respectiva actividad judicial, tanto ante el magistrado como ante el juez, por lo que quedaba excluida la posibilidad de actuar a través de un representante.

Ningún texto del período de las legis actiones ofrece información sobre este punto, por lo que es necesario acudir a fuentes de época posterior, en particular a los manuales de Derecho romano escritos en época clásica o postclásica que, tratando la institución de la representación procesal, aborden su introducción histórica en el período de las legis actiones.

Como afirma Murga, «Gayo, único jurista que nos ha explicado detalladamente la representación, reconoce en sus Instituciones que tal posibilidad de litigar nomine alieno no era lícita en tiempos de las viejas acciones» $\left(1_{9} 83,85\right)$ :

4. 82: Cum olim, quo tempore legis actiones in uso fuissent, alieno nomine agere non liceret...

El principio es confirmado por Ulpiano en su libro XIV ad edictum dedicado a los testamentos inoficiosos y a la querela inofficiosi testamenti:

D. 50. I7. I23 pr. (Ulpianus, XIV ad edictum): Nemo alieno nomine lege agere potest.

Vemos, pues, como en las legis actiones se produce una identidad entre los sujetos de la relación jurídica sustancial y los de la relación jurídica procesal. Asume la cualidad de parte aquél que afirmaba ser titular de la relación jurídica sustancial deducida en juicio y fundamento de la 
demanda (actor) y aquél que se oponía frente a tal demanda (demandado). No existe ningún desplazamiento de la legitimación procesal.

No explica Gayo el por qué de esta imposibilidad. En opinión de Murga «la razón habría que buscarla en la propia evolución del proceso romano. Todo el litigio no es en realidad más que una lenta transformación de la antigua lucha en donde las vis primitiva pasó con el tiempo, sin dejar de ser violenta, a convertirse en una 'lucha reglada' con sus palabras fijas y sus gestos rituales. Finalmente aunque, con el paso de los siglos, esta vieja lucha ordálica se transforme procesalmente en un verdadero juicio, la arcaica base conceptual nunca superada de dos personas en lucha, actúa de tal modo como condicionante, que sigue siendo poco menos que inconcebible el imaginar que pueda acudir al litigio una persona distinta del interesado» (1983, 86).

A pesar del rigor absoluto con el que Gayo presenta la imposibilidad agere nomine alieno, inesperadamente añade a continuación que no obstante en ciertos casos excepcionales, praeterquam ex certis causis ${ }^{I}$, se toleraba esta situación. A esas excepciones se refiere explícitamente un pasaje de las Instituciones de Justiniano ${ }^{2}$, seguramente relativo al proceso per legis actiones, aunque no se hace de ello mención alguna, y otro de la Paráfrasis de Teófilo, que recuerdan los casos: pro populo, pro libertate, pro tutela y ex lege Hostilia, todos ellos muy debatidos en la doctrina:

I. 4. Io pr.: Nunc admonendi sumus agere posse quemlibet aut suo nomine aut alieno.... cum olim in usu fuisset alterius nomine agere non posse nisi pro populo, pro libertate, pro tutela. Praeterea lege Hostilia permissum est furti agere eorum nomine, qui apud hostes essent aut reipublicae causa abessent, quive in eorum cuius tutela essent...

Paraphr. 4. I0:....... Scire oportet posse nos iudicio agere aut per nosmet ipsos, idest nostro nomine, aut per alios, idest alieno nomine. Et alieno nomine agimus, veluti ut procuratores tutores vel curatores: ex iure nimirum, quod hodie optinet. Olim enim in usu non erat alieno nomine agere, nisi ex certis causis; ut ecce pro populo quis litem inire poterat, aut pro libertate. Nam cum quis in possessione esset servitutis et in libertate proclamaret, non poterat ipse agere, ne forte, lite contestata, servus esse probaretur et fieret ut servus reapse cum domino ageret. Et ideo alius pro eo iudicium subibat, qui dicebatur adsertor. Item etiam pro tutela. Saepius enim fiebat, ut pupillus tutore indigeret et duo contenderent uter tutelam suscipere deberet. Patet alterutrum vincere debuisse. Qui itaque victus fuerat alieno nomine egisse videbatur, pro tutela nempe, quae ad eum non pertinebat, sicuti litis finis ostendit. Lex Hostilia permisit, ut si eorum, qui apud hostes essent vel qui reipublicae causa abessent, res ablatae estae essent, furti, qui vellet, ageret. Sed et si tutor fuissem pupilli, qui me apud hostes constituto reive publicae causa absente, furtum esset passus, aeque qui volebat, furti ei actio erat.....(Luzzatto I948, 275 s.).

I) La representación «pro populo» no es explicada en las Instituciones de Justiniano ni en la Paráfrasis de Teófilo. Parece que se litigaba pro populo en todos aquellos casos en que se ejercitaban acciones en interés de la comunidad. Como afirma Murga, «claramente se comprende que, por pura necesidad material, en estos litigios en favor o en contra de la organización estatal,

I Karlowa, observa que con anterioridad a la lectura del manuscrito de Verona realizada por Studemund, se había aceptado que Gayo mencionaba dos excepciones: nisi pro populo et libertatis causa (Karlowa 1872, 354).

2 No creemos que se pueda compartir la conclusión de Wirbel, de que el agere nomine alieno tiene en los dos textos un significado distinto (I9II, I8 y ss.) Coincide en su primera parte con Gayo 4.82: «Nunc admonendi sumus agere nos aut nostro nomine aut alieno». Además, el «olim» de I. 4.Io pr. equivale al «olim, quo tempore legis actiones in usu fuissent» de Gayo 4.82.

Sobre las fuentes consultadas por los compiladores a fin de completar esa pequeña laguna de la obra gayana , Ferrini (1929, 413) y Scialoja (I954, I90), afirman que habría sido las res cotidianae del mismo Gayo. Cfr. Arias Ramos observa que «no deja de haber una cierta contradicción entre tal conjetura y la afirmación del mismo Ferrini de que 'le res cotidianae di Gaio offrono una esposizione elementare del diritto privato, che si puo considerare como una nuova elaborazione delle Instituzioni, meno ricca di notizie storiche e di dettagli intorno a instituzioni antiquate, ma piú completa nella esposizione teorica del diritto vigente e nelle pratiche applicazioni'» (I938, 48 nt. 2I). Para Arias Ramos (I938, 48-49) y Albanese (I993, 20 nt. 45), no cabe descartar algún manuscrito de su Instituta con variantes en la redacción respecto al de Verona. 
aunque ésta se encontrara todavía en el estado embrionario propio de los primeros siglos, no habría otro camino útil que la representación si se deseaba actuar procesalmente. Sin embargo, hemos de entender -no en vano están Gayo y Justiniano estudiando el ordo iudiciorum privatorum- que estos representantes no tenían que ser necesariamente los cónsules ni ningún otro magistrado cualificado, sino cualquier ciudadano que lo deseara, qui volet ex populo» (1983, 87).

Se litigaba pro populo en todos aquellos casos en los que cualquier ciudadano estaba legitimado para reclamar la multa con que se castigaba al contraventor de una ley. Esta legitimación abierta a cualquier ciudadano que quisiera litigar, quicumque agere volet, probablemente ideada a fin de estimular con ello el celo de los magistrados, era precisamente lo que convertía en populares a estas acciones. En tales supuestos, el particular actúa posiblemente como si lo hiciera en representación de la colectividad y la multa se pronuncia en provecho de ésta, aun cuando se otorgue al particular, como premio, una participación en ella. Es posible que se refieran a esta hipótesis algunas inscripciones en donde se puede comprobar que era posible ejecutar con carácter popular una vía ejecutiva, como la manus iniectio para reclamar la multa con la que se castigaba ciertas contravenciones en materia de policía como, por ejemplo, el depositar inmundicias en determinados lugares o bien el enterrar o hacer sacrificios funerarios en lugares sagrados, según nos informa la inscripción de Luceria (Girard-Senn I967, $\mathrm{n}^{\circ}$ 25); o la manus iniectio y la pignoris capio, concedidas con fin semejante por el Senatus Consultum de pago montano (Riccobono I968, 272 y ss.).

Otro caso en el que se ha visto un agere pro populo, es aquél en el que cualquier ciudadano ejercita una legis actio sacramento in rem para reivindicar en nombre e interés de la comunidad la propiedad de un bien público poseído indebidamente por otro ciudadano. La prueba de la existencia de esta legis actio parece haberse encontrado en un pasaje del gramático Festo, que en la voz vindiciae afirma que a veces praetores secundum populum vindicias dicunt. Puesto que con el término vindiciae venía indicada, siempre según Festo, el objeto controvertido y ya que el dicere vindicias significaba la atribución, por orden del pretor, de la posesión interina de la cosa misma a uno de los litigantes, se afirma que eran casos en los cuales tal atribución venía a favor del populus romanus.

Estamos, en terminología de Bruns ${ }^{3}$ (I864, 24I y Ss.; I88I, 3 I3 y SS.), ante verdaderas acciones procuratorias donde la situación del demandante como representante aparece más clara frente a las acciones populares propiamente dichas o en sentido estricto. En estas el ciudadano demanda en su propio nombre y por su propia cuenta; el actor acciona en interés del pueblo, pero no lo hace como procurador del pueblo. En ellas existe un interés personal lesionado que a su vez era colectivo ya que coincidía con el de otros muchos ciudadanos que hubieran podido igualmente ejercitar la acción. Se sigue en su tramitación el procedimiento privado ordinario, y el importe de la multa que reclama va a parar a su patrimonio particular.

En todo caso, en este aspecto, suscribimos las palabras de Wenger cuando escribe «tuttavia la nostra conoscenza di queste cose è ancora esposta a parecchi dubbi» (1938, 83).

2) La representación «pro libértate» es ilustrada en la Paráfrasis. Responde a la incapacidad del esclavo para actuar procesalmente ante los órganos jurisdiccionales, bien como actor, bien como demandado. Claramente se comprende que en todos los casos en los que fuera discutido si un hombre es libre o es esclavo, se hacía necesaria la presencia de una tercera persona -adsertor libertatis- que le representase en la tramitación de la causa liberalis. La necesidad del adsertor libertatis viene impuesta tanto si se trata de una vindicatio ex servitute in

3 Vid. también Fadda (I894). Una sucinta exposición de lo esencial de la tesis de Bruns, y bibliografía sobre la materia, en Costa (I9I8, 53 nt. 2). Son interesantes los más recientes estudios de Casavola (I955, I3I y ss.); Idem (I958); Lozano Corbí (I982). 
libertatem, como si se trata de la vindicatio ex libertate in servitutem. En el primer caso, no presentándose ningún adsertor, aquél que de hecho es esclavo no tiene modo alguno de alegar las razones contrarias de su pretendida situación, por lo que el magistrado le consideraría indefensus y conserva su condición de esclavo; en el segundo caso, aquel que de hecho es libre, pero cuya condición viene discutida, se encontraría en la posición de ductus como consecuencia de la vindicatio in servitutem.

Como ha observado Arias Ramos, «en el aspecto doctrinal la situación del adsertor ofrece un matiz curioso, porque si su tesis resultaba victoriosa en el proceso, es obvio su papel de representante; pero si la causa liberalis se falla en contra suya, la idea de una representación se contradice con el carácter de objeto y no de sujeto de derecho que tiene el declarado esclavo y en cuyo nombre actuaba el adsertor. Sería entonces como un representante provisional de una persona presunta. Aunque la idea de la personalidad del servus desde el punto de vista del ius naturale podría resolver o suavizar la cuestión teórica.

Para el mencionado autor, la tarea representativa del adsertor comenzaba en momento distinto según que el estado en que de hecho viviese su representado fuese de esclavitud o de libertad. En el primer caso empezaba ya en el momento extrajudicial de la in ius vocatio; en el segundo caso en el acto in iure de proceder a la contravindicatio in libertatem, porque en la primera hipótesis el adsertor obraba como demandante y citaba a aquel que tenía como esclavo en su poder a su representado, mientras que en la segunda, el que pretendía tener el dominio sobre el hombre que de hecho vivía como libre obraba como demandante y, en cualquier lugar donde lo encontrase, podía forzar al que según él era esclavo a presentarse ante el magistrado para proceder a la vindicatio in rem praesentem» $(\mathrm{I} 938,5 \mathrm{I})$.

En el derecho justinianeo se admitió que el esclavo pueda actuar, en estas cuestiones, personalmente, ya sea como demandante ya sea como demandado; y que, en las vindicationes ex libertate in servitutem, el libre cuyo estado esta en cuestión pueda defenderse por medio de un procurator 5 .

3) No es fácil saber a qué caso pudo referirse el «agere pro tutela». La única explicación que aparece en las fuentes corresponde a la suministrada por la Paráfrasis griega de las Instituciones:

4 Vid. también Ferenczy (I973, 387 y ss.). Fernández de Buján, A., considera que «este caso probablemente haya constituido el más antiguo caso de representación judicial» $(2010,82)$.

5 C. J. 7. I7. I pr.(Imp. Iustinianus A. Mennae P.P.): Lites super servili conditione movendas as clementiorem tam examinationem quam terminum transferimus, iubentes, si quis vel adhuc serviens liberum se esse dixerit, vel in libertate commorans ad servitutem vocatus fuerit, assertoris difficultatem in utroque casu cessare, ipsum que per se ad intentiones eius, qui dominum sese asserit, respondere et si es possessione libertatis ad servitutem ducitur,, etiam procuratorem dare minime prohiberi, quod his, qui ex servitute ad libertatem prosiluerint, penitus interdicimus; illis legibus, quae dudum et secunda et tertia vice assertorias lites examinari praecipiebant, in posterum conquiescentibus, quum sit iustum, primam definitionem in suis manere viribus, quum provocatio nulla oblata fuerit. Qua porrecta, ad similitudinem aliorum negotiorum iudex, ad quem res ex provocatione ducitur, eam examinabit, cuius et ipsius iudicium ad secundam exquisitionem minime deducetur occasione legum, quae super assertoriis litibus positae sunt. I.- Super peculio etiam eorum vel aliis rebus aut causis veterem defensoris observationem tollimus, praecipientes illorum tantummodo peculia, qui ex possessione servitutis super libera conditione litigant, aliasque res, quae vindicantur, in tuto pro dispositione iudicis collocari. 2.- Omnes vero, qui pro libertate periclitantur, si quidem possint fideiussorem dare, eum exigi; sin vero revera datio eius impossibilis eis sit, hocque iudici manifeste ostendatur, iuratoriae cautioni committi; scientes quod, si post huiusmodi expositionem abfuerint, et edictis citati in absentia nihilominus per unum annum duraverint, omnimodo servituti obnoxii erunt, et eius dominio, qui litem eis intulit, sine ulla dubitatione assignabuntur. 3.- Scire vero eos volumes, qui aliquem ad servitutem vocant, quod, si post primam accusationem in quocunque iudicio vel ex divali iussione factam, et admonitionem ei oblatam, qui servus esse dicitur, in alio eum accusaverint etsi domini sint, suo iure privabuntur. 
4. Io pr.: Item etiam pro tutela. Saepius enim fiebat, ut pupillus tutore indigeret et duo contenderent uter tutelam suscipere deberet. Patet alterutrum vincere debuisse. Qui itaque victus fuerat alieno nomine egisse videbatur, pro tutela nempe, quae ad eum non pertinebat, sicuti litis finis ostendit.

Para Teófilo se da un agere pro tutela cuando dos personas discuten entre sí sobre quién de ellos es el verdadero tutor del pupilo: aquél que pierde el litigio habría actuado alieno nomine, en cuanto que habría accionado sobre la base de una tutela que no le correspondía, habrá seguido un pleito pro tutela sin ser tutor ${ }^{6}$.

Descartada la interpretación de Teófilo, la opinión mayoritaria considera el agere pro tutela como la actuación del tutor en interés del pupilo ${ }^{7}$. Sin embargo, no han dejado de presentarse objeciones a tal interpretación ${ }^{8}$. Así, Murga considera que «en primer lugar, el tutor nunca litiga en nombre del pupilo sino en nombre propio y por sí mismo, como si fuese realmente el dueño de los bienes pupilares hasta el momento en que el tutelado salga de la tutela y él mismo deba rendir cuenta de su administración. Por otra parte, si ese fuese el caso no se diría pro tutela sino pro pupilo ya que sería exactamente el pupilo y no la tutela quien se encontraría bajo esa actuación procesal» $(1983,87)$. Añadimos que de admitir la opinión mayoritaria, el agere pro tutela se constituiría en una excepción de las, a su vez, otras tres excepciones al principio de la no representación procesal en el sistema de las legis actiones, lo que parece encajar difícilmente en la idea común de legitimación popular que preside dichas excepciones.

Ante tales objeciones, otro sector de la doctrina considera como la interpretación más probable aquella ya formulada por Rumelin (I886, 348 y ss.) y Hruza (I887, 67 y ss.) ${ }^{9}$, que centraban la actuación lege agere pro tutela en el caso de conflicto de intereses entre el tutor y el pupilo y, en particular, en el caso de la accusatio suspecti tutoris. Esta interpretación es quizá la única que supera el obstáculo constituido por la naturaleza popular de la primitiva representación procesal y además atribuye a la palabra «pro tutela» un sentido sustancialmente análogo a aquél de los otros tres casos, así como entender el mismo en su significado más llano y literal de actuar en favor de la tutela, entendida como instituto.

6 Scialoja ha dado la siguiente explicación, generalmente aceptada por la doctrina, al supuesto error de Teófilo: «Existía, especialmente en el derecho justinianeo, una actio protutelae, dada contra quien había gestionado como tutor, sin serlo verdaderamente, los asuntos del pupilo; o sea, contra el falsus tutor. No es improbable pues, que Teófilo, o quien fuese el autor de la Paráfrasis, al leer pro tutela en su fuente, hubiese evocado la actio protutelae, y como en ésta hay un falsus tutor, hubiera imaginado un caso en que compareciera un tutor que no fuera tal contra el verdadero tutor». Scialoja ofrece otra explicación, muy hipotética, del posible error de Teófilo, relativa a una confusión con el tutor praetorius, de la que da noticia Gayo, I.I84: «En tiempo de las legis actiones había un tutor nombrado expresamente para dar su auctoritas en favor del pupilo o de la mujer y contra el tutor; teníamos allí una litis de un tutor contra otro tutor; y acaso también esta reminiscencia de una litis entre dos tutores (y aquí, evidentemente, el tutor pretorio nombrado al efecto accionaba alieno nomine y el tutor ordinario lo hacía suo nomine), puede haber dado lugar al error de Teófilo» (I954, I9Iy ss.). Buonamici considera que se trata del tutor pretorio (I886, 539). Cfr. Cosentino afirma que el tutor praetorius no era representante, sino sólo asistía, merced a la auctoritatis interpositio, al pupilo «qui fari potest» en un proceso contra el efectivo tutor que, a causa del evidente conflicto de intereses, no habría podido ser auctor in rem suam (ig64, 275 nt. 37).

7 Participan de esta interpretación, entre otros autores, Eisele (I88I, 53 y ss.); Girard (I90I, 70 nt. I; 97 nt. I); sin embargo en Manuel, habla de «cas incertain» ( I978, I089); Scialoja (I954,I92 y ss.); Bertolini (I9I3, I87); Costa (I9I8, I2I); Wenger (I938, 83); Arias Ramos (I938, 51 y ss.); Âlvarez Suárez (I955, 2I7); Orestano (I967, 797); Provera (I989, 334); Scapini (I992, 205); Albanese (I993, 20 y ss.); Kaser (I996, 63); Fernández de Buján, A. (2010, 82).

8 Vid., Karlowa (1872, 355); Cosentino (1964, 263 y ss.); Murga (1983, 87). Cfr. la defensa que algunos de los autores citados en la nota anterior hacen frente a tales críticas: Bertolini (I9I3, I87 y ss.); Scialoja (I954, I92 Ss.); Arias Ramos (I938, 52 Ss.); Albanese (I993, 2I nt. 53).

9 Comparten esta interpretación, Wirbel (I9II, I3 y nt. I); Luzzatto (1948, 278 y ss.); Cosentino (I964, 263 y ss.); Murga (1983, 87). 
Dicha hipótesis, sin embargo, presenta el problema de la tramitación de la postulatio suspecti tutoris. Como es sabido, su tramitación se realiza a través de un procedimiento acusatorio particular, más concretamente por una cognitio praetoria, que el magistrado decide mediante decreto. Si ello es así, el agere pro tutela constituiría una excepción procedimental respecto a los otros tres casos de representación procesal mencionados en Inst. 4. Io pr., pues estos se tramitan mediante una legis actio. Cosentino (I964, 278 y ss.), para salvar el contenido del agere pro populo, piensa que ni Gayo ni Justiniano han querido hacer una referencia específica al modus procedendi de la época primitiva, sino una referencia puramente cronológica a dicho período. Los compiladores en Inst. 4, Io pr. habrían recogido como casos de agere nomine alieno aquellas acciones que tienen una legitimación directa universal y en el ámbito de éstas habrían incluido, indiferentemente, acciones que se tramitan mediante un lege agere y acciones que se desarrollan procedimentalmente de otra forma, por ejemplo, a través de procedimientos de naturaleza pública.

4) El último caso de representación judicial de la época de las legis acciones enumerado en las Instituciones, y único explicado en ellas, es el de la representación judicial «ex lege Hostilia», permitía que cualquier ciudadano romano pudiese acudir al litigio en nombre de un absens rei publicae causa. Originariamente la representación debía de estar limitada únicamente a favor del que se encuentra prisionero de los enemigos, ampliándose más tarde a otras ausencias por motivos políticos. La interpretación jurisprudencial extendió este supuesto inicial al caso de un impúber sometido a tutela, cuando fuera precisamente su tutor quien se encontrase en las situaciones mencionadas anteriormente. La intervención del representante a favor del ausente parece haberse limitado al ejercicio de la condictio furtiva.

No ha podido determinarse la fecha de la lex Hostilia. Huvelin la relacionaba con diversos plebiscitos del siglo VII de Roma (I9I5, II7); Rotondi atribuye su creación a C. Hostilius Tubulus, pretor urbano del año 547 a. C. (I9I2, 480); en todo caso, como afirma Arias Ramos «parece probable que la acción creada por esta ley sea la más moderna de las excepciones al principio nemo nomine alieno agere potest del procedimiento de las acciones de la ley» $(\mathrm{I} 938,53)$.

En opinión de Arias Ramos, «se trata de una de esas acciones populares, en el sentido más amplio de esta denominación, de aquellas que quilibet ex populo puede promover. Es una de las que -según Bruns- sólo los comentaristas modernos llaman populares, ya que los romanos reservaban esta calificación -dentro de las que quilibet ex populo puede ejercitar- a las establecidas iuris publici tuendi gratia, en las que ius suum populo tuentur, y las ventajas de cuya sentencia favorable pertenecían al actor» $(1938,53)$.

\section{El cognitor. su existencia y su esfera de actividad en época de las legis actiones}

Constituye una cuestión polémica en la doctrina romanística, quizá por el silencio que guardan las fuentes, la relativa a si existió y, en caso afirmativo, con qué esfera de actividad, el cognitor en época de las legis actiones.

Los autores mantienen opiniones diversas: en favor de la tesis negativa se pronuncian Bethmann-Hollweg (I865, 4I7 nt. 47), Wlassak (I892, I y ss.), Mayr (I926, II3), Wenger (I938, 82 y ss.), Scialoja (1954, I93 y ss.), Murga (I983, 88 y ss.), Lozano (I999, 290, 32I) o Fernández de Bujan, A. (20I0, 83). Por el contrario, defienden la idea apuntada por Schmidt y Huschke de que el cognitor se utilizó en el período de las legis actiones, entre otros, Karlowa (I872, 356 y ss.), Wirbel (I9II, I y ss., 23 y ss.), Bertolini (I9I3, I88 y ss.), Costa (I9I8, I22 y ss.), Arias Ramos (I938, 53 y ss.), Luzzatto (I948, 282 y ss.), Álvarez Suárez (I955, 217 y nt. I53), Pugliese (I963, 326 nt. 6I), Gómez-Iglesias (I984, 20), Albanese (I993, 21 y ss.), Fernández de Buján, F (I994, 4I8, 423 y nt. 
27, 436 y ss.), duda Kaser (I996, 63), no obstante sus discrepancias acerca del momento procesal en el que aparece funcionando.

El hecho de que Justiniano no enumere al cognitor como excepción al principio alieno nomine agere non liceret, hace que los partidarios de la tesis negativa, volviendo los ojos hacia el testimonio literal de Gayo, afirmen que en tiempos de las viejas acciones de la ley no se dio realmente, salvo los casos excepcionales anteriormente vistos, ningún tipo de representación procesal voluntaria. En nuestra opinión, su silencio puede tener otra interpretación. La Instituta, aunque destinada a la enseñanza, fue promulgada como ley, razón que abunda en la omisión de una figura histórica totalmente anacrónica para los compiladores. Ello viene corroborado por el propio tenor literal de Inst. 4, Io pr., donde los compiladores consideran al procurador como el primer representante procesal, en clara inarmonía histórica con Gayo 4. 83. Y sin embargo, sorprenden dos de las razones que a continuación dan los compiladores y que justificarían su nombramiento: la enfermedad y la edad, razones que curiosamente, como veremos más adelante, son las aducidas por el autor de la Rethorica ad Herennium para justificar la designación del cognitor.

Otro argumento infravalorado por los partidarios de la tesis negativa, que apoya la idea de que el cognitor se utilizó en el período de las legis actiones, a nuestro juicio muy verosímil, son las diversas consideraciones que permiten extraer la observación de los caracteres de las fórmulas de designación de este representante procesal que Gayo nos transmite $(4,83)$ : a) las palabras sacramentales y solemnes -certis verbis- que recuerdan la rigidez formalista de las legis actiones y, en todo caso, son un indicio cierto del origen bastante remoto de la figura; b) el hecho de que su nombramiento no tolerase diem vel condicionem ${ }^{\text {I0 }}$ y que hace que venga considerado entre los actus legitimi, y c) la necesidad de que estén presentes ambas partes litigantes -coram adversario-, inmediatez típica en todos los negocios y figuras jurídicas de la antigüedad.

A pesar de que parece probable que la figura del cognitor ya era conocida en el período de las legis actiones, sigue siendo un misterio el determinar la posible fecha en que fue introducido. El único dato existente es una frase contenida en la Rethorica ad Herennium (2. I3. 20), compuesta entre los años 86 y 82 a. C., y atribuida durante mucho tiempo a Cicerón: «ut maior annis LX et cui morbus causa est cognitorem det». Los autores que niegan la existencia del cognitor en el sistema de las legis actiones consideran esta referencia «muy imprecisa» y «relativamente tardía como para que su testimonio sobre el origen del cognitor pudiera sernos útil»y, por supuesto, la refieren al proceso formulario ${ }^{\text {II }}$. Sin embargo, creemos que cabe otra lectura. Si el anónimo tratado retórico se refiere al proceso formulario, es difícil explicar por qué se limita la representación procesal del cognitor a esos dos casos, cuando en dicho proceso tiene una aplicación general. Más verosímil resulta la idea de los partidarios de la tesis positiva de referirlo a la época de las legis actiones, no antes de la mitad del siglo IV de Roma. Además, la mención que la Rethorica hace del cognitor, omitiendo por el contrario al procurator, no es fácilmente explicable referida al procedimiento formulario, pero sí lo es históricamente si la referimos a las legis actiones.

La referencia de la Rethorica ad Herennium, nos permite delimitar el ámbito de actuación del cognitor en el proceso de las legis actiones a los casos en que el actor o demandado fuese mayor de sesenta años o estuviese impedido o enfermo, es decir, casos de absoluta imposibilidad material de asistir al proceso. Interpretación, que vendría a completar y no a sustituir el fragmento de las XII Tablas (I. 3) que ordenaba al demandante: «si morbus aevitasve vitium scit,

IO Frag.Vat. 3I8(Ulp. VIII ad ed.): Non tamen sic putat certis verbis cognitorem dari debere, ut, si quid fuisset adiectum vel detractum, non valeat datio ut in legis actionibus. Frag. Vat. 329(Pap. II resp.): Sub condicione cognitur non recte datur, non magis quam mancipatur aut acceptum vel expensus fertur: nec ad rem pertinet, an ea condicio sit inserta, quae non expressa tacite inesse videatur.

II Murga (1983, 89) 
iumentum dabo», no en el sentido atribuido por Eisele (I884, I94 nt. I) de que la ley decenviral se refiere a la fase in iure, mientras que la alusión de la Rethorica lo hace a la fase in iudicio, sino como dos medidas tendentes a lograr la comparecencia de las partes -in iure y apud iudicem-y presididas por una idea de equidad ${ }^{\mathrm{r2}}$.

De nuevo son las Instituciones de Gayo la fuente principal para conocer las formalidades de designación del cognitor:

4, 83: Cognitor autem certis verbis in litem coram adversario substituitur. Nam actor ita cognitorem dat: QUOD EGO A TE verbi gratia FUNDUM PETO, IN EAM REM LUCIUM TITIUM TIBI COGNITOREM DO; adversarius ita: QUIA TU A ME FUNDUM PETIS, IN EAM REM TIBI PUBLIUM MEVIUM COGNITOREM DO. Potest ut actor ita dicat: QUOD EGO TECUM AGERE VOLO, IN EAM REM COGNITOREM DO, adversarius ita: QUIA TU MECUM AGERE VIS, IN EAM REM COGNITOREM DO. Nec interest presens an absens cognitor detur; sed si absens datus fuerit, cognitor ita erit, si cognoverit et susceperit officium cognitoris.

Su nombramiento se realiza pronunciando ciertas palabras sacramentales y solemnes certis verbis-, delante de la otra parte litigante -coram adversario- y asume el proceso en nombre propio $^{\text {I3 }}$.

Se discute si era preceptivo que la designación se hiciera ante el magistrado. Es significativo el silencio que guarda Gayo sobre este extremo, principal argumento para aquellos que no creen en tal requisito. Quizá, como afirman Bertolini ((I9I4, 263 nt. 3) y Arias Ramos (1938, 63), la semejanza e íntima conexión del procurator apud acta factus con el cognitor, juntamente con la necesidad de la constitución de aquél in iure, induce a admitir como necesaria la presencia del magistrado.

Gayo, además, nos dice en el texto reproducido, que no era indispensable que el cognitor estuviera presente en el momento de su nombramiento y que si se hallaba ausente, era necesario notificárselo, y que lo aceptara de modo expreso, si bien existe alguna duda sobre este punto por su contradicción con otros textos:

Festo, $\mathrm{v}^{\circ}$ cognitor: «cognitor est, qui litem alterius suscipit coram eo cui datum est».

Isidoro, Differ. I, I23: «cognitor non nisi praesens a praesente praesenti datun».

Interpretatio ad C. Th. 2, I2, de cogn. et procur., 7: «cognitor est, cui sine mandato causam suam agendam praesens praesente iudice litigator iniungit».

Ante tal disparidad no resulta fácil descubrir cuál pudo ser en realidad la verdad histórica. Sin embargo, la mayoría de los autores, creemos que con razón, han preferido atenerse al testimonio de Gayo. Arias Ramos afirma que «ha de darse un mayor valor al texto gayano, que, como informador del derecho clásico, tiene que merecer un crédito superior al de testimonios tan distanciados de la época como San Isidoro y los autores de la Interpretatio en la compilación romano-visigoda. Por lo que hace a la definición de Festo, pertenece a aquella parte de su obra que nos es trasmitida por el epítome de Paulo el Diácono y existen dudas de que éste recogiese de un modo fidedigno la redacción de Sexto Pomponio Festo». Para Luzzatto «l'assenza del cognitor si riferisca soltanto al secondo dei due esempi prospettati da Gaio: quando, cioè, non siamo

I2 Wirbel (I9II, 24 y nt. 2; 33 nt. I; Gómez-Iglesias (I984, 20 y nt. I3).

I3 Scialoja observa que «la palabra cognitor, además de indicar un particular representante judicial, adopta el significado de perito; así en la ley malacitana c. 63-65 se habla de cognitores llamados a responder de la valoración de los fundos dados en garantía al municipio por los contratistas» (I954, I97). Bertolini, añade que también dicho término es usado por el juez, en el derecho avanzado del Imperio, especialmente en materia penal (I9I4, 270 nt. 3). Vid. también Wirbel (I9II, VII nt. I). 
ancora in sede di litis constestatio. Ciò in quanto, altrimenti, non si vede come, in quest'ultimo caso, il ocgnitor potrebbe contestare la lite in proprio» (I938, 64). Murga (1983, 9I nt. 87) afirma que «tal vez Gayo tenga razón y el dato que el obispo hispano-romano nos aporta sea producto de un pequeño error entre el cognitor y el llamado procurator praesens, forma tardía de procurator, nombrado sin solemnidad y liberado de la obligación de prestar cauciones. No han faltado autores que han tratado de conciliar los testimonios de Gayo y San Isidoro». En opinión de Bertolini «certamente è da preferire l'asserto di Gaio; non escludendo tuttavia che in una fase primitiva si sia richiesta la praesentia anche del cognitor costituendo ed in seguito si sia decampato da tale requisito» (I9I4, 27I nt. 2).

El nombramiento del cognitor venía considerado entre los actus legitimi que no admiten condición ${ }^{\mathrm{T}}$. Probablemente tampoco el término podría adicionarse a la cognitoris datio teniendo en cuenta lo que se dispone en D. 50, I7, 77, donde, naturalmente, los compiladores suprimieron la referencia a dicho acto $^{15}$.

Según Gayo existían dos fórmulas para designar al cognitor, sin embargo el jurista no nos aclara en qué casos se empleaba una de ellas y no la otra. Partiendo de la idea de que no es verosímil que, indistinta y caprichosamente, en cualquier hipótesis y con idénticos efectos, se pudiera elegir indiferentemente, se ha buscado la explicación en el examen de los términos en que las mismas están redactadas.

Savigny $\left(\mathrm{I} 846,306\right.$ ss.) y Buonamici $(\mathrm{I} 886,54 \mathrm{I})^{\mathrm{I} 6}$ consideran que la primera fórmula (... fundum peto...) serviría para ejercitar acciones reales, mientras que la segunda (... agere volo...) para ejercitar acciones personales. Bethmann-Hollweg (1865, 4I7 nt. 50), Wlassak (I892, 25 y ss.) y Scialoja (I954, I96) ${ }^{17}$ suponen que la primera fórmula se utilizaría para designar a un cognitor para un determinado proceso, designación que sería simultánea con la litis contestatio y, por tanto, tal representante actuaría ante el juez -in iudicio-, no ante el magistrado -in iure-; la otra fórmula se emplearía para instituir un cognitor para un futuro proceso. Karlowa (I872, 359 nt. 2), Eisele (I88I, 28 y ss.), Bertolini (I9I4, 27I, 274 nt. 4) y Gehrich (I963, 6 y ss.) ${ }^{\text {I8 }}$ consideran que originariamente sólo existiría la primera fórmula, la segunda habría surgido más tarde con la atenuación del riguroso formalismo del ius civile, momento a partir del cual podrían utilizarse ambas indistintamente. En todo caso, parece indudable que las palabras empleadas por el poderdante en una y otra fórmula, indican que la designación se hace en ambos casos en distintos momentos del proceso. Esta interpretación es mantenida por Wirbel (I9II, Ioo y ss.), Wenger (I938, 83), Arias Ramos (I938, 66 y ss.), Luzzatto (1948, 283 y ss.), Pugliese (I963, 327), o Álvarez Suárez (I955, 218 nt. I54) y entienden que la primera fórmula debía ser usada para nombrar al cognitor en el momento de la litis contestatio o posterior a ésta, ya en la fase in iudicio, mientras que la segunda fórmula se utilizaría antes de la litis contestatio, durante la fase in iure o en el momento de la edictio actionis extraprocesal. Esta interpretación ha sido matizada por Zablocka

\footnotetext{
I4 Fr. Vat. 329 (Pap. I. 2 resp.): Sub condicione cognitor non recte datur, non magis quam mancipatur aut acceptum vel expensum fertur...

I5 D. 5 O. I7. 77 (Pap. 38 Quaest.): Actus legitimi, qui non recipiunt diem vel conditionem, veluti mancipatio, acceptilatio, hereditatis aditio, servi optio, datio tutoris, in totum vitiantur per temporis vel conditionis adiectionem ....

I6 Cfr. Wirbel afirma por lo que se refiere concretamente a petere también es utilizado en la actio in personam (I9II, IO2 nt. 3).
}

I7 Cfr. Pugliese, observa, con toda razón, que «ogni cognitor sembra essere costituito solo per una determinata lite e, in ogni caso, solo di fronte ad un determinato avversario, che deve essere presente» (I963, 327 nt. 62).

I8 Cfr. Pugliese afirma que «è facile osservare, in contrario, che per la dichiarazione di nomina -elemento esenziale del formulario- entrambi gli schemi prevedevano la medesima expressione -cognitorem do-» (I963, $327 \mathrm{nt}$. 62). 
(I983-84, I44, I48) en el sentido de que la verdadera editio actionis, con significado procesal, tiene lugar en el momento de la in ius vocatio, por lo tanto, en un proceso judicial ya iniciado.

Naturalmente, se comprende que en todos los casos anteriormente examinados, el representante actúa movido por un interés distinto al económico, por lo que su actuación procesal no genera gasto alguno. Así, en el caso pro populo, afirma Murga que «esta actuación supone toda una manera típicamente romana de concebir los deberes cívicos, al permitir que cualquier ciudadano pueda litigar haciendo realmente suyo el interés comunal» (1983, 87). En el agere pro tutela, escribe el citado autor que «alguien que actúa en nombre de otro y además sin ser tutor sino simplemente un cives que, sintiendo como suya esta situación inicua, interviene legítimamente en el proceso sin ser realmente el interesado directo». Dentro de la concepción romana del officium $^{19}$ o deber de solidaridad que tiene el hombre hacia sus propios conciudadanos se enmarcan las actuaciones pro libertate, ex lege Hostilia y la del cognitor, lo mismo que sucede con otros tipos de officia como en el officium iudicis, parentis, mariti, tutoris, etc.

El officium como expresión de solidaridad, introducido por la filosofía estoica, se difunde en Roma ejerciendo una profunda influencia sobre la sociedad romana tanto bajo el aspecto ético como jurídico $^{2 \circ}$. En palabras de Cremades «la existencia de una cierta correspondencia entre la moral estoica y las tradicionales virtudes de los romanos - mos maiorum- es un hecho. Virtudes como la gravitas, la constantia, la magnitudo animis, la pietas y la fides, actitudes como el espíritu de trabajo y la simplicidad de la vida, todas ellas nobles y ancestrales tradiciones, se reencuentran, en cierta manera, en el ideal moral de los estoicos. En el cumplimiento del officium radica el vivir el altruismo, la solidaridad con los demás, el humanitarismo. Lo que cuenta es la disposición del hombre hacia los demás; la actitud servicial y benéfica, consecuencia del conocimiento del propio deber; conocimiento que está en dependencia del conocimiento de lo que es otro es» (I988, 43 y ss.).

\section{Bibliografía}

ALBANESE (I993): Il processo privato romano delle legis actiones, Palermo.

ÁLVAREZ SUÁREZ (I955): Curso de Derecho Romano, Madrid.

ARIAS RAMOS (1938): «La representación procesal en el Derecho Romano», Boletín de la Universidad de Santiago de Compostela 28, p. 4I y ss.

BERNERT (1930): De vi atque usu vocabuli officii, Breslau.

BERTOLINI (I9I3): Appunti didattici di Diritto Romano. Il processo civile, II, 1, Torino.

BERTOLINI (I9I4): Appunti didattici di Diritto Romano. Il processo civile, II, 2, Torino.

BETHMANN-HOLLWEG (I865): Der römische Civilprozess, vol. II, Bonn.

BRUNS (I864): «Die römischen Popularklagen», Zeitschrift für Rechtsgeschichte 3, p. 24I y ss.

BUONAMICI (I886): La storia della procedura civile romana, Pisa: ed.anast.

I9 No es muy abundante la bibliografía moderna sobre una normatividad tan interesante como la que supone el officium, con todo, son muy completos los estudios de Bernet (I930, reed. 2000, 40, 44, I70, I73, I8I, 256); Cancelli (I957-58, 35I y ss.); Idem, (I960, 229 ss.); Cervenca (I968, I53 y ss.); Murga (I978, II6 y ss.); Cremades (I988); Iglesias (I99I, 52 y ss.); Coppola (I994, 2II y Ss.).

20 Para la influencia del pensamiento estoico sobre los jurisconsultos, la bibliografía es amplísima. Vid., entre los autores más recientes, Coing (I952, 24 y ss.); Idem (I953, 365 y ss.); Wieacker (I953, 93 y ss.); Idem (I96I, I43 y ss.); Ormanni (I962, 596 y ss.); Miquel (I970); Idem (I977); Grosso (I976, I39 y ss.); Talamanca (I977, 3 y ss.); Reinoso (I988). 
CANCELLI (I957-58): «Saggio sul concetto di officium en diritto romano», Rivista italiana per le scienze giuridiche 9, p. 35I y ss.

- (I960): «Nota preliminare sull 'officium civile’», St. Vassalli vol. I, p. 229 ss.

CASAVOLA (I955): «Fadda e la dottrina delle azioni popolari», Labeo. Rassegna di diritto romano I, p. I3I y ss.

CASAVOLA (1958): Studi sulla azioni popolari, Napoli.

CERVENCA (I968): «Sull'uso del termino officium nella legislazione postclassico-giustinianea», St. Grosso vol. III, p. I53 SS.

COING (I952): «Zum Einfluss der Philosophie des Aristóteles auf die Entwicklung des römischen Rechts», Zeitschrift der savigny-Stiftung für Rechtsgeschichte 69, p. 24 y ss.

COING (I953): «Zur Methodik der republikanischen Jurisprudenz: Zur Entstehung der grammatisch logischen Auslegung», St. Arangio-Ruiz vol. I, p. 365 y ss.

COPPOLA (I994): Cultura e potere. Il lavoro intellattuale nel mondo romano, Milano.

COSENTINO (I964): «Sul 'pro tutela agere' di Inst. 4, Io pr.», Studia et Documenta Historiae Iuris 30, p. 270 y ss.

COSTA (I9I8): Profilo storico del processo civile romano, Roma.

CREMADES (1988): El officium en el derecho privado romano. Notas para su estudio, León.

DE SIMONE (1967): s.v. «Rapresentanza in giudizio», NNDI I4, p. 857 y ss.

EISELE (188I): Cognitor und Procurator: Untersuchungen zur Geschichte der processialen Stellvertretung, Tübingen.

EISELE (I884): «Zur ältesten cognitur», Zeitschrift der savigny-Stiftung für Rechtsgeschichte 5, p. I94 y ss.

FADDA (I894): L'azione popolare, Roma.

FERENCZY (I973): «L'<Adsertor Libertatis> nell'etá della Repubblica romana arcaica», Studi in memoria di Donatuti vol. I, p. 387 y ss.

FERNÁNDEZ DE BUJÁN, F. (I994): «Notas acerca del origen del contrato de mandato y su relación con la procuraduría en Derecho romano», Boletín de la Facultad de Derecho de la UNED 7, p. 418 y ss.

FERNÁNDEZ DE BUJÁN, A. (20I0): Derecho privado romano, Madrid.

FERRINI (I929): Sulle fonti delle Instituzioni di Giustiniano, Opere, II, Milano.

GEHRICH, W.D. (I963): Kognitur und Prokuratur in rem suam als Zessionsformen des klassischen römischen Rechts, Gotinga.

GIRARD (I9OI): Histoire de l'organisation judiciaire des romains. Les six premiers siècles de Rome, Paris.

GIRARD-SENN (I967): Textes de Droit Romain, Paris.

GÓMEZ-IGLESIAS (I984): Citación y comparecencia en el procedimiento formulario romano, Santiago de Compostela.

GROSSO (I976): «Influenze aristoteliche sulla sistemazione delle fonti delle obligación nella giurisprudenza romana», La filosofia greca e il diritto romano. Coloquio italo-frances vol. I, p. I39 y ss

HRUZA (I887): Ueber das lege agere pro tutela, Erlangen.

HUVELIN (I9I5): Études sur le furtum dans les très ancien romain, vol. I, Paris.

IGLESIAS (I99I): «Officium», Espíritu del derecho romano.

KARLOWA (I872): Der römische Civilprozess zur zeit der Legisactionen, Berlín.

KASER (1996): Das römische ZivilprozessRecht, München.

LOZANO CORBÍ (I982): La legitimación popular en el proceso romano clásico, Barcelona.

LOZANO CORBÍ (I999): Historia e Instituciones de Derecho Romano, Zaragoza.

LUZZATTO (I948): Procedura civile romana. Le legis actiones, Bologna.

MAYR (I926): Historia del Derecho Romano, vol. I, trad. de W. Roces, Barcelona.

MIQUEL (I970): «Stoische Logik und römische Jurisprudenz», Zeitschrift der savigny-Stiftung für Rechtsgeschichte 87.

MIQUEL (I977): Aenigma, La Laguna.

MURGA (I978): «Un original concepto de officium en Séneca (Epist. I02. 6)», Anuario de Historia del Derecho español 48 , p. II6 y ss. 
MURGA (1983): Derecho Romano Clásico. El Proceso, Zaragoza.

ORESTANO (1967): s.v. «Rapresentanza», NNDI i4, p. 797.

ORMANNI (Ig62): «Penus legata. Contributi alla storia dei legati disposti con clausola penale in età republicana e clásica», St. Betti vol. IV, p. 596 y ss

PROVERA (I989): Lezioni sul processo civile giustinianeo I-II, Torino.

PUGLIESE (1963): Il processo civile romano. II. Il processo formulare, I, Milano.

REINOSO (I988): «La autonomía de la jurisprudencia romana frente al pensamiento filosófico griego», Estudios homenaje al Prof. Juan Iglesias vol. II.

RICCOBONO (1968): Fontes Iuris Romani Antejustiniani, Florentiae.

ROTONDI (1912): Leges Publicae Populi Romani, Milano.

RUMELIN (I886): Geschichte der Stellvertretung in römischen Civilprozess, Freiburg.

SAVIGNY (I846): «Kritische Bemerkungen zum Vierten Buch der Institutionem des Gaius», Zeitschrift für geschichtliche Rechtswissenschaft $\mathrm{I} 3$, p. 306 y ss.

SCAPINI (I992): Istituzioni di diritto romano, Parma.

SCHULZ (2000): Principios del derecho romano, trad. Abellán, Madrid.

SCIALOJA (1954): Procedimiento civil romano, trad. Sentis Melendo y Ayerra Redin, Buenos Aires.

TALAMANCA (I977): «Lo schema genus-species nelle sistematiche dei giuristi romani», La filosofia greca e il diritto romano vol. II, p. 3 y ss

WENGER (I938): Istituzioni di procedura civile romana, trad. ital. Orestano, Milano.

WIEACKER (I953): «Griechische Wurzeln des Institutionem-systems», Zeitschrift der savigny-Stiftung für Rechtsgeschichte 70 , p. 93 y ss.

WIEACKER (I96I): Vom römischen Recht, vol. II, Stuttgart.

WIRBEL (I9II): Le cognitor, París.

WLASSAK (I892): «Zur Geschichte der Cognitur», Festgabe zum Doktor-Jubilüum R. Ihering, p. I y ss.

ZABLOCKA (I983-84): «La costituzione del 'cognitor' nel processo romano clásico», en INDEX I2, p.I40 y ss.

\section{Abreviaturas}

\section{FUENTES}

C. J. - Codex Iustiniani.

D. - Digesta.

Frag. Vat. - Fragmenta Vaticana.

GAYO - Gaius, Institutiones.

I. - Institutiones Iustiniani.

NNDI - Novissimo Digesto Italiano

Paraphr. -Paraphrasis de Teófilo. 